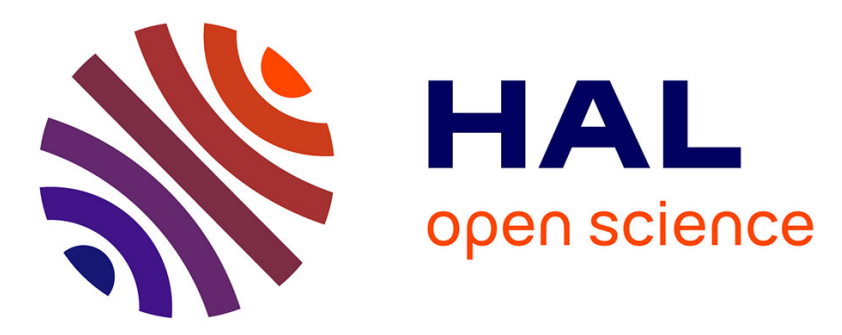

\title{
Stability of discontinuous homogeneous nonlinear sampled-data systems
}

\author{
Emmanuel Bernuau, Emmanuel Moulay, Patrick Coirault
}

\section{To cite this version:}

Emmanuel Bernuau, Emmanuel Moulay, Patrick Coirault. Stability of discontinuous homogeneous nonlinear sampled-data systems. Automatica, 2019, 107, pp.467-473. 10.1016/j.automatica.2019.06.013 . hal-02266749

\section{HAL Id: hal-02266749 \\ https://hal.science/hal-02266749}

Submitted on 23 Dec 2020

HAL is a multi-disciplinary open access archive for the deposit and dissemination of scientific research documents, whether they are published or not. The documents may come from teaching and research institutions in France or abroad, or from public or private research centers.
L'archive ouverte pluridisciplinaire HAL, est destinée au dépôt et à la diffusion de documents scientifiques de niveau recherche, publiés ou non, émanant des établissements d'enseignement et de recherche français ou étrangers, des laboratoires publics ou privés. 


\title{
Stability of discontinuous homogeneous nonlinear sampled-data systems *
}

\author{
Emmanuel Bernuau $^{\mathrm{a}}$, Emmanuel Moulay $^{\mathrm{b}}$, Patrick Coirault ${ }^{\mathrm{c}}$ \\ ${ }^{a}$ GENIAL (UMR INRA 1145), AgroParisTech, 1 Avenue des Olympiades, 91744 Massy Cedex, France \\ ${ }^{\mathrm{b}}$ XLIM (UMR CNRS 7252), Université de Poitiers, 11 Bd Marie et Pierre Curie, 86962 Futuroscope Chasseneuil Cedex, France \\ ${ }^{\mathrm{c}}$ LIAS (EA 6315), ENSIP Poitiers, 2 rue Pierre Brousse, 86073 Poitiers Cedex 9, France
}

\begin{abstract}
The article deals with the stability of discontinuous homogeneous nonlinear systems with aperiodic sampled-data inputs. We first extend results about robustness of homogeneous nonlinear systems to the discontinuous case. Then, we prove that this framework is well suited for studying the stability of discontinuous homogeneous nonlinear sampled-data systems. The results are illustrated on the double integrator.
\end{abstract}

Key words: Sampled-data systems, Differential inclusions, Homogeneity, Lyapunov stability, Lyapunov functions.

\section{Introduction}

The problem of stability for systems with sampled-data inputs has been renewed with the development of networked control systems [1]. Due, for instance, to wireless communications, the quality of the channel between the controller and the actuator can be insufficient to ensure a quick updating of the feedback control. This leads to the problem of knowing if the stability properties pertain through sampled-data inputs [2]. Lot of works have been done to solve this problem such as the input/output approach [3] or the sum of squares approach [4]. Moreover, several different modeling alternatives as hybrid systems $[1,5]$, discrete-time systems [6] or time-delay systems [7-10] have also been used to tackle this problem. Most of these strategies $[3,4,11]$ are developed under the emulation method where the controller is first designed in continuous time and then implemented as a sampleddata controller $[12,13]$.

The notion of homogeneity was introduced in $[14,15]$

\footnotetext{
‡ This paper was not presented at any IFAC meeting. Corresponding author E. Bernuau. Tel. +33 (0)1 699350 83; Fax +33(0)169935020.

Email addresses: emmanuel. bernuau@agroparistech.fr (Emmanuel Bernuau), emmanuel.moulay@univ-poitiers.fr (Emmanuel Moulay), patrick.coirault@univ-poitiers.fr (Patrick Coirault).
}

and developed for instance in [16-18]. Moreover, stability theorems for perturbed homogeneous systems were first studied in [19]. Recently, we have developed in [20] a new nonlinear emulation strategy based on homogeneity which uses the input-to-state stability (ISS) properties of homogeneous systems in order to preserve their stability properties when they are subject to aperiodic sampled-data inputs. This strategy has been developed in the framework of continuous controllers but we can wonder if it still works in the case of discontinuous controllers. Homogeneous properties of discontinuous systems have been developed for instance in [21]. Recently, the ISS properties of homogeneous systems have been extended to discontinuous systems in [22]. Some papers have also stated robustness properties of discontinuous homogeneous systems (see for instance [23]). However, no robustness condition allowed us to assess stability properties for emulated discontinuous homogeneous systems.

To highlight the contribution of this paper, we would like to point out that, although systems controlled by discontinuous control laws are well known to present robustness properties with respect to exogenous perturbations (like external forces acting on the system), it is also well known that, when subject to endogenous perturbations (like sampling), these systems may present bad behaviors, chattering being the most famous. To understand the difference, let us present a simple scalar ex- 
ample showing the difficulties appearing when the continuity is lost. Consider the system $\dot{x}=-\mid x+\delta\rceil^{\alpha}$, with $x \in \mathbb{R}$ and $\alpha \geq 0$, where $\delta$ is an unknown disturbance and $\lfloor z\rceil^{\alpha}=\operatorname{sign}(z)|z|^{\alpha}$. A commonly used technique consists in writing $\lfloor x+\delta\rceil^{\alpha}=\lfloor x\rceil^{\alpha}+\left(\lfloor x+\delta\rceil^{\alpha}-\lfloor x\rceil^{\alpha}\right)$ which gives the effect of the nominal system $-\lfloor x\rceil^{\alpha}$, added to a perturbative term $\Delta=\lfloor x+\delta\rceil^{\alpha}-\lfloor x\rceil^{\alpha}$. We would like the perturbative part to be dominated by the nominal part in order to bring the system to 0 . When $\alpha>0$ the system is continuous and we can show that $|\Delta| \leq 2|\delta|^{\alpha}$, which implies that $\Delta \rightarrow 0$ when $\delta \rightarrow 0$ uniformly in $x$. When $\alpha=0$ we reach the discontinuous case and then $\Delta=0$ when $|\delta|<|x|$ and \pm 2 elsewhere. We still have $\Delta \rightarrow 0$ when $\delta \rightarrow 0$ but this convergence is not uniform anymore, leading to a cascade of technical problems to prove the stability. Obviously, in this very specific and very simple example, solutions can be found by hand; but in the general case we need specific techniques to address the discontinuous case.

In this paper, we prove that, under well chosen technical assumptions, discontinuous homogeneous systems behave nicely when subject to endogenous perturbations, in particular samplings. The difficulty was to find a condition, namely Assumption 15, that would then allow us to use the same techniques than in [20], while not being too constraining to apply to a large set of systems. Moreover, the results fall into the framework of sliding mode control which has been used for sampled-data systems in [24-26]. Homogeneous sliding mode controllers has been proposed recently in [27].

The article is organized as follows. After some preliminaries stated in Section 2, we develop a new result about the robustness of homogeneous systems in Section 3. Then we use this result in the context of homogeneous sampled-data systems for addressing the main result of the article in Section 4. A numerical application to the double integrator is given Section 5. Concluding remarks appears in Section 6.

\section{Preliminaries}

\subsection{Mathematical notation}

Throughout the paper the following notation is used:

- $\mathbb{R}_{+}=\{x \in \mathbb{R}: x \geq 0\}$, where $\mathbb{R}$ is the set of real numbers.

- $|\cdot|$ denotes the absolute value in $\mathbb{R},\|$.$\| denotes the$ Euclidean norm on $\mathbb{R}^{n}$

- $B(y, \varepsilon)=\left\{x \in \mathbb{R}^{n}:\|x-y\|<\varepsilon\right\}$ and $\bar{B}(y, \varepsilon)$ is its closure.

- If $r_{1}, \ldots, r_{n} \in \mathbb{R}$, we will denote $\operatorname{Diag}\left(r_{1}, \ldots, r_{n}\right)$ the diagonal matrix of dimension $n \times n$ with $k^{t h}$ diagonal entry $r_{k}$.

- For a (Lebesgue) measurable function $d: \mathbb{R}_{+} \rightarrow \mathbb{R}^{n}$, we denote $\|d\|_{[a, b]}=\operatorname{ess}_{\sup } \operatorname{sia,b]}_{t}\|d(t)\|$. If for any $0 \leq$ $a<b$ we have $\|d\|_{[a, b]}<+\infty$, the function $d$ is called locally essentially bounded. We denote $\mathcal{L}_{\text {loc }}^{\infty}$ the set of locally essentially bounded functions $d: \mathbb{R}_{+} \rightarrow \mathbb{R}^{n}$.

- A continuous function $\alpha: \mathbb{R}_{+} \rightarrow \mathbb{R}_{+}$belongs to the class $\mathcal{K}$ if $\alpha(0)=0$ and the function is strictly increasing. A function $\alpha: \mathbb{R}_{+} \rightarrow \mathbb{R}_{+}$belongs to the class $\mathcal{K}_{\infty}$ if $\alpha \in \mathcal{K}$ and it is increasing to infinity.

- A continuous function $\beta: \mathbb{R}_{+} \times \mathbb{R}_{+} \rightarrow \mathbb{R}_{+}$belongs to the class $\mathcal{K} \mathcal{L}$ if $\beta(\cdot, t) \in \mathcal{K}_{\infty}$ for each fixed $t \in \mathbb{R}_{+}$ and if for each fixed $s \in \mathbb{R}_{+}$the function $t \mapsto \beta(s, t)$ is decreasing to 0 .

- The notation $d_{x} V$ (resp. $\left.d_{x} \Phi\right)$ stands for the differential of the function $V$ (resp. the diffeomorphism $\Phi$ ) at the point $x$.

\subsection{Differential inclusions}

In this subsection, we recall the basic concepts of differential inclusions and the Filippov procedure. For details and proofs, see [28].

Definition 1 A set-valued map $F: \mathbb{R}^{n} \rightrightarrows \mathbb{R}^{n}$ is upper semicontinuous (USC) if for any $x \in \mathbb{R}^{n}$ and any neighborhood $\mathcal{V}$ of $F(x)$, there exists a neighborhood $\mathcal{U}$ of $x$ such that for any $y \in \mathcal{U}, F(y) \subset \mathcal{V}$.

Definition 2 Let $F: \mathbb{R}^{n} \rightrightarrows \mathbb{R}^{n}$ be a set-valued map. We say that $F$ verifies the standard assumptions if $F$ is $U S C$ and if for any $x \in \mathbb{R}^{n}, F(x)$ is a nonempty compact convex set.

Definition 3 Let $g: \mathbb{R}^{n} \times \mathbb{R}^{m} \rightarrow \mathbb{R}^{n}$ be locally essentially bounded. We denote $\mathcal{F}_{x}[g](x, \Delta)$ the set

$$
\bigcap_{\varepsilon>0} \bigcap_{N \in \mathcal{N}} \overline{\operatorname{conv}}\{g(y, \Delta): y \in B(x, \varepsilon) \backslash N\}
$$

where $\mathcal{N}$ denotes the set of all Lebesgue-negligible subsets of $\mathbb{R}^{n}$. The set-valued map $\mathcal{F}_{x}[g]:(x, \Delta) \mapsto \mathcal{F}_{x}[g](x, \Delta)$ is called the Filippov's regularization of $g$.

Proposition 4 Let $g: \mathbb{R}^{n} \times \mathbb{R}^{m} \rightarrow \mathbb{R}^{n}$ be locally essentially bounded. For any $\Delta \in \mathbb{R}^{m}$, the set-valued map $G_{\Delta}: \mathbb{R}^{n} \rightrightarrows \mathbb{R}^{n}$ defined by $G_{\Delta}(x)=\mathcal{F}_{x}[g](x, \Delta)$ verifies the standard assumptions.

We refer to [28] for the definitions of a differential inclusion and the corresponding notions of solutions, convergence and stability.

\subsection{Input-to-State Stability}

Let us consider the following nonlinear system:

$$
\dot{x}=f(x, d),
$$


where $x \in \mathbb{R}^{n}$ is the state, $d \in \mathcal{L}_{\text {loc }}^{\infty}$ is the external input and $f: \mathbb{R}^{n+m} \rightarrow \mathbb{R}^{n}$ is continuous. We will also denote $f_{d}(x)=f(x, d)$ and hence $f_{0}(x)=f(x, 0)$.

Let us introduce the definition of input-to-state stability given for instance in [29].

Definition 5 The system (2) is called input-to-state stable (ISS), if for any input $d \in \mathcal{L}_{\text {loc }}^{\infty}$ and any $x_{0} \in \mathbb{R}^{n}$ there exist some functions $\beta \in \mathcal{K} \mathcal{L}$ and $\gamma \in \mathcal{K}$ such that for any solution $x(t)$ of the equation (2) we have:

$$
\|x(t)\| \leq \beta(\|x(0)\|, t)+\operatorname{ess}^{\sup _{[0, t]}} \gamma(\|d\|) \quad \forall t \geq 0 .
$$

The function $\gamma$ is called a nonlinear asymptotic gain.

\subsection{Homogeneity}

Different notions of homogeneity appear in the litterature. The most common one is the weighted homogeneity, based on a particular choice of the coordinates, while the most generic one is the geometric homogeneity, which is coordinate free. We shall more use in the sequel the framework of geometric homogeneity than the framework of weighted homogeneity, so we will introduce the latter as a particular case of the former.

Definition 6 A vector field $\nu$ on $\mathbb{R}^{n}$ is called an Euler vector field if $\nu$ is of class $C^{1}$, complete (i.e. the maximal integral curves are defined on $\mathbb{R}$ ) and if the origin is a globally asymptotically stable equilibrium of $-\nu$.

Example 7 If we consider a matrix $A \in \mathbb{R}^{n \times n}$ such that $-A$ is Hurwitz, the vector field defined by $\nu(x)=A x$ is an Euler vector field. In particular, if $A=\operatorname{Diag}\left(r_{1}, \ldots, r_{n}\right)$ with $r_{1}, \ldots, r_{n}>0$, the vector field $\nu(x)=A x$ is Euler.

Definition 8 Let $\nu$ be an Euler vector field on $\mathbb{R}^{n}$. We denote $\Phi^{s}(x)$ the value of the flow of $\nu$ at time $s$ with initial condition $x$.

- A function $V: \mathbb{R}^{n} \rightarrow \mathbb{R}$ is $\nu$-homogeneous of degree $\kappa \in \mathbb{R}$ if for all $x \in \mathbb{R}^{n}$ and all $s \in \mathbb{R}$ we have $V\left(\Phi^{s}(x)\right)=e^{\kappa s} V(x)$.

- A vector field $f$ on $\mathbb{R}^{n}$ is $\nu$-homogeneous of degree $\kappa \in$ $\mathbb{R}$ if for all $x \in \mathbb{R}^{n}$ and all $s \in \mathbb{R}$ we have $f\left(\Phi^{s}(x)\right)=$ $e^{\kappa s} d_{x} \Phi^{s} f(x)$.

Remark 9 If we have $\nu(x)=A x$, with $-A$ Hurwitz, the flow of $\nu$ verifies $\Phi^{s}(x)=\exp (A s) x$. In particular, if $A=\operatorname{Diag}\left(r_{1}, \ldots, r_{n}\right)$ with $r_{1}, \ldots, r_{n}>0$, we find $\Phi^{s}(x)=\operatorname{Diag}\left(e^{r_{1} s}, \ldots, e^{r_{n} s}\right) x$. The homogeneity defined by such an Euler vector field has been the subject of a lot of works. It is usually referred to as weighted homogeneity, the coefficients $r_{1}, \ldots, r_{n}$ are called the weights and $\mathbf{r}=\left[r_{1}, \ldots, r_{n}\right]$ is called the generalized weight [30]. Let us finally mention that homogeneity with respect to an Euler vector field defined by a generalized weight $\mathbf{r}$ is usually simply referred to as r-homogeneity.
Equivalent conditions for homogeneity exist. Given that we will not need them below, we just refer to $[16,17]$ for a complete exposition of the homogeneity theory.

Let us now introduce a tool and two lemmas useful in the sequel.

Definition 10 Let $\nu$ be an Euler vector field on $\mathbb{R}^{n}$. A $\nu$-homogeneous norm is a function $N: \mathbb{R}^{n} \rightarrow \mathbb{R}$ such that:

(1) $N$ is positive definite;

(2) $N$ is $\nu$-homogeneous of degree 1 ;

(3) $N$ is continuous.

Lemma 11 Let $V: \mathbb{R}^{n} \rightarrow \mathbb{R}$ be a continuous positive definite $\nu$-homogeneous function of degree $\kappa>0$. There exist $\sigma_{-}$and $\sigma_{+}$two functions of class $\mathcal{K}_{\infty}$ such that for all $x \in \mathbb{R}^{n}$, we have $\sigma_{-}(\|x\|) \leq V(x) \leq \sigma_{+}(\|x\|)$.

Proof. Denote $\sigma_{+}(r)=\sup _{\|x\| \leq r} V(x)$. The function $\sigma_{+}$ is clearly continuous, increasing and verifies $\sigma_{+}(0)=0$. Let us show that $\sigma_{+}$is strictly increasing. It is enough to prove that for any $x_{0}$ such that $\left\|x_{0}\right\| \leq r$ and $V\left(x_{0}\right)=$ $\sigma_{+}(r)$ verifies $\left\|x_{0}\right\|=r$. Assume by contradiction that $\left\|x_{0}\right\|<r$. By continuity of the flow $\Phi$, there exists $\varepsilon>0$ such that for all $s \in\left[0, \varepsilon\left[\right.\right.$, we have $\left\|\Phi^{s}\left(x_{0}\right)\right\|<r$ and thus $V\left(\Phi^{s}\left(x_{0}\right)\right) \leq \sigma_{+}(r)=V\left(x_{0}\right)$. But $V\left(\Phi^{s}\left(x_{0}\right)\right)=$ $e^{\kappa s} V\left(x_{0}\right)>V\left(x_{0}\right)$ for $s>0$, which is a contradiction. Hence we get that $V(x) \leq \sigma_{+}(\|x\|)$. Now, given that, for $x \neq 0,\left\|\Phi^{s}(x)\right\| \rightarrow+\infty$ when $s \rightarrow+\infty$, we see that $e^{\kappa s} V(x)=V\left(\Phi^{s}(x)\right) \leq \sigma_{+}\left(\left\|\Phi^{s}(x)\right\|\right)$ and finally by taking the limit in both sides we get $\lim _{r \rightarrow+\infty} \sigma_{+}(r)=+\infty$, that is $\sigma_{+} \in \mathcal{K}_{\infty}$.

The function $\sigma_{-}$is defined by $\sigma_{-}^{-1}(r)=\sup _{V(x) \leq r}\|x\|$. We similarly prove that $\sigma_{-}^{-1} \in \mathcal{K}_{\infty}$. Finally, $V(x) \leq$ $\sup _{\|y\| \leq\|x\|} V(y)=\sigma_{+}(\|x\|)$ and $\|x\| \leq \sup _{V(y) \leq V(x)}\|y\|=$ $\sigma_{-}^{-1}(V(x))$, that is $V(x) \geq \sigma_{-}(\|x\|)$.

Lemma 12 [23] Let $\sigma: \mathbb{R}_{+} \rightarrow \mathbb{R}$ be an increasing function such that $\lim _{x \rightarrow 0^{+}} \sigma(x)=0$. Then there exists $a$ class $\mathcal{K}$ function $\bar{\sigma}$ such that for all $x \in \mathbb{R}_{+}, \sigma(x) \leq \bar{\sigma}(x)$.

\section{Robustness of discontinuous homogeneous systems}

We consider the following differential inclusion, depending on a parameter $\Delta$ :

$$
\dot{x} \in F(x, \Delta), \quad x \in \mathbb{R}^{n}, \Delta \in \mathbb{R}^{n} .
$$

We make the following assumptions on the system (3).

Assumption 13 The origin is globally asymptotically stable for the system (3) with $\Delta=0$. 
Assumption 14 There exists $\kappa<0$ and a matrix $A \in$ $\mathbb{R}^{n \times n}$ such that $-A$ is Hurwitz and such that for all $x \in$ $\mathbb{R}^{n}$, all $\Delta \in \mathbb{R}^{n}$ and all $s \in \mathbb{R}$ we have

$$
F\left(e^{A s} x, e^{A s} \Delta\right)=e^{\kappa s} e^{A s} F(x, \Delta) .
$$

Assumption 15 For all compact set $X \subset \mathbb{R}^{n}$, there exists $\sigma_{X} \in \mathcal{K}$ such that for all $x \in X$ and all $\Delta \in \mathbb{R}^{n}$

$$
F(x, \Delta) \subset F(x+\Delta, 0)+\bar{B}\left(0, \sigma_{X}(\|\Delta\|)\right) .
$$

The following result is an extension to discontinuous systems of Theorem 11 in [31].

Theorem 16 Under Assumptions 13, 14 and 15, the system (3) is ISS w.r.t. the input $\Delta$. Moreover, if we denote $\nu(x)=A x$ and if $N$ is any $\nu$-homogeneous norm, then there exists constants $\alpha>0, b>0$ and $C>0$ such that for any solution $x(t)$ of $(3)$ :

$$
N(x(t)) \leq \alpha \max \{\beta(N(x(0)), t) ; C N(\Delta)\},
$$

where

$$
\beta(r, t)=\max \left\{r^{-\kappa}-b t, 0\right\}^{-\frac{1}{\kappa}} .
$$

Proof. Given Assumption 13, Theorem 3.22 in [32] gives us a Lyapunov pair $(V, W)$ of class $C^{1}, \nu$-homogeneous functions of degree $\mu>0$ and $\mu+\kappa>0$ respectively such that for all $x \neq 0$ we have $V(x)>0, W(x)>0$ and for all $v \in F(z, 0)$ we have $d_{x} V v \leq-W(x)$. Let $x \neq 0$ be fixed and, given $\Delta \in \mathbb{R}^{n}$, let us pick $v \in F(x, \Delta)$. We denote $N=V^{1 / \mu}, \mathbb{S}=\{N=1\}$ and $s=\ln (N(x))$, i.e. $N(x)=e^{s}$. It yields that $\bar{x}=e^{-A s} x \in \mathbb{S}$. We define $\bar{\Delta}=e^{-A s} \Delta$ and $\bar{v}=e^{-\kappa s} e^{-A s} v$. Assumption 14 gives us that $\bar{v} \in e^{-\kappa s} e^{-A s} F(x, \Delta)=F(\bar{x}, \bar{\Delta})$. Hence we get $d_{x} V v=\left(e^{\mu s} d_{\bar{x}} V e^{-A s}\right)\left(e^{\kappa s} e^{A s} \bar{v}\right)=e^{(\mu+\kappa) s} d_{\bar{x}} V \bar{v}$. Following Assumption 15 with $X=\mathbb{S}$, there exists $w \in$ $F(\bar{x}+\bar{\Delta}, 0)$ and $r \in \bar{B}\left(0, \sigma_{\mathbb{S}}(\|\bar{\Delta}\|)\right)$ such that $\bar{v}=w+r$. It yields

$$
d_{x} V v=e^{(\mu+\kappa) s}\left[d_{\bar{x}+\bar{\Delta}} V w+\left(d_{\bar{x}} V-d_{\bar{x}+\bar{\Delta}} V\right) w+d_{\bar{x}} V r\right]
$$

Now, defining

$K(\rho)=\left\{\varpi \in \mathbb{R}^{n}: \exists \delta \in \bar{B}(0, \rho), \exists z \in \mathbb{S}, \varpi \in F(z+\delta, 0)\right\}$

and $M(\rho)=\sup \{\|\varpi\|: \varpi \in K(\rho)\}$ we find that, since $F(\cdot, 0)$ is USC, $M(\rho)$ is finite for any $\rho \geq 0$. Moreover, $M$ is increasing. Upper bounding the three parts of the right-hand side of (6) yields

$$
\begin{aligned}
d_{\bar{x}+\bar{\Delta}} V w & \leq-W(\bar{x}+\bar{\Delta}) \\
& \leq-\inf _{K(\|\bar{\Delta}\|)} W \\
\left(d_{\bar{x}} V-d_{\bar{x}+\bar{\Delta}} V\right) w & \leq M(\|\bar{\Delta}\|) \sup _{z \in \mathbb{S}}\left\|d_{z} V-d_{z+\bar{\Delta}} V\right\| \| \\
d_{\bar{x}} V r & \left.\leq \sup _{z \in \mathbb{S}}\left\|d_{z} V\right\| \sigma_{\mathbb{S}}(\|\bar{\Delta}\|)\right)
\end{aligned}
$$

For $\bar{\Delta}$ small enough, we have $K(\|\bar{\Delta}\|) \subset \mathbb{R}^{n} \backslash\{0\}$ and hence $\inf _{K(\|\bar{\Delta}\|)} W>0$. Moreover, $\rho \mapsto-\inf _{K(\rho)} W$ is increasing and therefore there exists $a>0$ and $\eta_{1}>0$ such that if $\|\bar{\Delta}\|<\eta_{1}$ then $-\inf _{K(\|\bar{\Delta}\|)} W<-a$.

The continuity of $d V$ shows that there exists $\eta_{2}>0$ such that if $\|\bar{\Delta}\|<\eta_{2}$, then $M(\|\bar{\Delta}\|) \sup _{z \in \mathbb{S}}\left\|d_{z} V-d_{z+\bar{\Delta}} V\right\| \mid<$ $\frac{a}{4}$. The continuity of $\sigma_{\mathbb{S}}$ shows that there exists $\eta_{3}>0$ such that if $\|\bar{\Delta}\|<\eta_{3}$, then $\left.\sup _{z \in \mathbb{S}}\left\|d_{z} V\right\| \sigma_{\mathbb{S}}(\|\bar{\Delta}\|)\right)<\frac{a}{4}$. Finally we find that, denoting $\eta=\min \left\{\eta_{1}, \eta_{2}, \eta_{3}\right\}$, if $\|\bar{\Delta}\|<\eta$ then $d_{x} V v<-e^{(\mu+\kappa) s} \frac{a}{2}<0$. Thus, following Lemma 11, there exists $C^{2}>0$ such that if $C N(\stackrel{\Delta}{\Delta})<1$ we have $d_{x} V v<0$. But $\bar{\Delta}=e^{-A s} \Delta$ and hence $N(\bar{\Delta})=e^{-s} N(\Delta)=\frac{N(\Delta)}{N(x)}$. We conclude that $d_{x} V v<-\frac{a}{2} V(x)^{\frac{\mu+\kappa}{\mu}}$ when $N(x)>C N(\Delta)$ which leads to (4) and (5) with $b=-\frac{a \kappa}{2 \mu}$. Finally, the constant $\alpha>0$ appears when taking another homogeneous norm.

Among the assumptions on which relies the Theorem 16, Assumption 13 is necessary when looking at ISS properties, Assumption 14 is a homogeneity-related assumption and Assumption 15 is a weak continuity assumption. Assumption 15 arises naturally when a continuous control system is controlled by a discontinuous feedback law as shown in the following lemma.

Lemma 17 Consider the following disturbed system

$$
\dot{x}=f(x, u(x+\Delta))
$$

with $f: \mathbb{R}^{n} \times \mathbb{R}^{m} \rightarrow \mathbb{R}^{n}$ continuous, $u: \mathbb{R}^{n} \rightarrow \mathbb{R}^{m}$ a locally essentially bounded state feedback and $\Delta \in \mathcal{L}_{\text {loc }}^{\infty}$. The set-valued map $F=\mathcal{F}_{x}[g]$, where $g(x, \Delta)=f(x, u(x+$ $\Delta)$ ), verifies Assumption 15.

Proof. The function $f$ is continuous, therefore for any compact set $X \subset \mathbb{R}^{n}$ the function $\varphi: \mathbb{R}_{+} \rightarrow \mathbb{R}_{+}$defined by

$$
\begin{array}{r}
\varphi(\rho)=\sup \{\|f(y+\Delta, u(y+\Delta))-f(y, u(y+\Delta))\|, \\
y \in X+\bar{B}(0,1),\|\Delta\| \leq \rho\}
\end{array}
$$

is increasing and $\varphi(0)=0$. The continuous function $f$ is uniformly continuous on the compact set $(X+\bar{B}(0,1)) \times$ $\bar{B}(0,1)$ and thus $\varphi$ is continuous at 0 . Lemma 12 yields that there exists a function $\sigma_{X} \in \mathcal{K}$ such that for all 
$\rho \geq 0, \varphi(\rho) \leq \sigma_{X}(\rho)$. Hence, for all $x \in X$ and all $y \in \bar{B}(x, 1)$ :

$$
\begin{aligned}
f(y, u(y+\Delta))= & f(y+\Delta, u(y+\Delta)) \\
& +f(y, u(y+\Delta))-f(y+\Delta, u(y+\Delta)) \\
\in & f(y+\Delta, u(y+\Delta))+\bar{B}\left(0, \sigma_{X}(\|\Delta\|)\right)
\end{aligned}
$$

which leads to $F(x, \Delta) \subset F(x+\Delta, 0)+\bar{B}\left(0, \sigma_{X}(\|\Delta\|)\right)$.

\section{Example 18 Consider the double integrator}

$$
\left\{\begin{array}{l}
\dot{x}_{1}=x_{2} \\
\dot{x}_{2}=u
\end{array}\right.
$$

We define the control law

$$
u(x)=-k_{1} \operatorname{sign} x_{1}-k_{2} \operatorname{sign} x_{2}
$$

with $k_{1}>k_{2}>0$. It is well-known that the origin is a globally finite-time stable equilibrium of the closed-loop system (9)-(10) [33], which yields Assumption 13. Taking $A=\operatorname{Diag}(2,1)$ we can easily check that Assumption 14 holds for the following disturbed system with $\kappa=-1$

$$
\left\{\begin{array}{l}
\dot{x}_{1}=x_{2} \\
\dot{x}_{2}=-k_{1} \operatorname{sign}\left(x_{1}+\Delta_{1}\right)-k_{2} \operatorname{sign}\left(x_{2}+\Delta_{2}\right)
\end{array}\right.
$$

Finally, from Lemma 17, Assumption 15 holds as well for the system (11) and finally Theorem 16 shows that there exists $b<0$ and $C>0$ such that, for any solution $x(t)$ of $(11)$ and any $[2,1]$-homogeneous norm $N$ :

$$
N(x(t)) \leq \max \{\beta(N(x(0)), t) ; C N(\Delta)\},
$$

where

$$
\beta(r, t)=\max \{r+b t, 0\} .
$$

\section{Sampled-data systems}

Consider the following control system

$$
\dot{x}=f(x, u)
$$

with $x \in \mathbb{R}^{n}, u \in \mathbb{R}^{m}$ and $f$ continuous. We suppose known a locally essentially bounded state feedback $u(x)$ such that the origin is a globally asymptotically stable equilibrium for the closed-loop system

$$
\dot{x}=f(x, u(x)) \text {. }
$$

We want to know how the system (13) reacts when subject to the sampling of the control law $u(x)$, that is when $u(x(t))$ is replaced by $u\left(x\left(t_{k}\right)\right)$ for a given sequence of instants $\left(t_{k}\right)_{k \in \mathbb{N}}$

$$
\dot{x}(t)=f\left(x(t), u\left(x\left(t_{k}\right)\right)\right), \quad t \in\left[t_{k}, t_{k+1}\right) .
$$

Denoting, for $t \in\left[t_{k}, t_{k+1}\right), \Delta(t)=x\left(t_{k}\right)-x(t)$, we find that the system (14) is equivalent to

$$
\dot{x}=f(x, u(x+\Delta)) .
$$

Given that $u$ is discontinuous, the vector field defined in (15) is discontinuous and we shall replace it with the differential inclusion coming from the Filippov's procedure. Hence defining $F=\mathcal{F}_{x}[g]$, where $g(x, \Delta)=$ $f(x, u(x+\Delta))$, we reformulate $(15)$ by

$$
\dot{x} \in F(x, \Delta) \text {. }
$$

Let us see now the consequences of Theorem 16 on the sampling effects.

Theorem 19 Consider a sequence of sampling times $\left(t_{k}\right)_{k \in \mathbb{N}}$ for which there exists $\eta, h \in \mathbb{R}$ such that $0<$ $\eta \leq t_{k+1}-t_{k} \leq h<+\infty$. Assume that the reformulated sampled differential inclusion (16) is such that Assumptions 13 and 14 hold. Consider $\nu(x)=A x$ and $N$ any $\nu$-homogeneous norm. Then, there exists $\xi>0$ such that the set $\mathcal{N}=\left\{x \in \mathbb{R}^{n}: N(x) \leq \xi h^{-1 / \kappa}\right\}$ is globally asymptotically stable w.r.t. the system (14).

The proof of Theorem 19 is largely inspired by the proof of Theorem 12 in [20]. A couple of technical details are however different, given the discontinuous nature of the system (13). For the sake of clarity, a complete proof is thus provided below.

Proof. The proof of the theorem is divided into 4 steps.

I. We show that it is sufficient to prove the result for a given $h$ when $\kappa \neq 0$.

II. We show that we can apply Theorem 16 .

III. We prove a preliminary fact on the increase rate of $N(x(t))$.

IV. We prove the theorem for $\kappa<0$.

Step $I$. When $\kappa \neq 0$, assume that the results of Theorem 19 hold for a given maximal sampling period $h_{0}>0$. Let us prove that the results then hold for any $h>0$. Indeed, consider $s \in \mathbb{R}$ such that $h=e^{-\kappa s} h_{0}$. Consider a solution $x(t)$ of (16) with $\left(t_{k}\right)_{k \in \mathbb{N}}$ the sequence of sampling times. Then consider $z(t)=\exp (A s) x\left(e^{\kappa s} t\right)$. If $t_{k} \leq e^{\kappa s} t<t_{k+1}$, we have

$$
\dot{z}(t) \in e^{\kappa s} \exp (A s) F\left(x\left(e^{\kappa s} t\right), x\left(t_{k}\right)-x\left(e^{\kappa s} t\right)\right) .
$$

From Assumption 14, it follows that

$$
\begin{aligned}
\dot{z}(t) & \in F\left(\exp (A s) x\left(e^{\kappa s} t\right), \exp (A s)\left(x\left(t_{k}\right)-x\left(e^{\kappa s} t\right)\right)\right) \\
& \in F\left(z(t), z\left(e^{-\kappa s} t_{k}\right)-z(t)\right) .
\end{aligned}
$$


Hence the curve $z(t)=\exp (A s) x\left(e^{\kappa s} t\right)$ is a solution of (16) with sampling times $\left(e^{-\kappa s} t_{k}\right)_{k \in \mathbb{N}}$. It follows that the mapping $x(t) \mapsto \exp (A s) x\left(e^{\kappa s} t\right)$ is a bijection between the solutions of (16) with sampling times $\left(t_{k}\right)_{k \in \mathbb{N}}$ and the solutions of (16) with sampling times $\left(e^{-\kappa s} t_{k}\right)_{k \in \mathbb{N}}$. Now, the local asymptotic stability for the maximal sampling period $h_{0}$ ensures the existence of $\beta_{0} \in \mathcal{K} \mathcal{L}$ such that

$$
N(x(t)) \leq \beta_{0}\left(N\left(x_{0}\right), t\right)+\xi h_{0}^{-1 / \kappa}, \quad \forall t \geq 0 .
$$

By the point (2) of Definition 8 , we have

$$
\begin{aligned}
N(z(t)) & =e^{s} N\left(x\left(e^{\kappa s} t\right)\right) \\
& \leq e^{s} \beta_{0}\left(N\left(x_{0}\right), e^{\kappa s} t\right)+e^{s} \xi h_{0}^{-1 / \kappa} \\
& \leq e^{s} \beta_{0}\left(e^{-s} N\left(z_{0}\right), e^{\kappa s} t\right)+\xi\left(e^{-\kappa s} h_{0}\right)^{-1 / \kappa} \\
& \leq \beta_{s}\left(N\left(z_{0}\right), t\right)+\xi h^{-1 / \kappa}
\end{aligned}
$$

with $\beta_{s}(r, t)=e^{s} \beta_{0}\left(e^{-s} r, e^{\kappa s} t\right) \in \mathcal{K} \mathcal{L}$.

Step II. The signal $\Delta$ is piecewise continuous and verifies $\dot{\Delta}=-f(x, \Delta)$ and $\Delta\left(t_{k}\right)=0$. It is straightforward to check that Assumptions 13 and 14 hold. Assumption 15 also holds from Lemma 17. The origin being a globally asymptotically stable equilibrium of the $\nu$-homogeneous system $\dot{x} \in F(x, 0)$ of degree $\kappa$, by virtue of Theorem 3.22 in [32], there exists a $\nu$-homogeneous smooth Lyapunov function $V$ of degree $\mu>0$ with $\mu+\kappa>0$. Let us define $N(x)=V(x)^{1 / \mu}$. We will prove the theorem for this particular homogeneous norm $N$, but Proposition 10 from [20] implies that the theorem then holds for all homogeneous norms.

Step III. Step II and Theorem 16 lead to

$N(x(t)) \leq \max \left\{\beta(N(x(0)), t) ; C \operatorname{ess} \sup _{\tau \in[0, t]} N(\Delta(\tau))\right\}$

with $C>0$ and $\beta(r, t)=\max \left\{r^{-\kappa}-b t, 0\right\}^{-1 / \kappa}$ (see the proof of Theorem 16). We define $\mathbb{S}=\left\{z \in \mathbb{R}^{n}: N(z)=\right.$ $1\}$ and for any $\varepsilon>0$

$\alpha(\varepsilon)=\sup \left\{\left\|d_{z} V v\right\|: V(z) \leq \varepsilon^{\mu}, y \in \mathbb{S}\right.$ and $\left.v \in F(y-z, z)\right\}$

and $H=\sup _{0<\varepsilon<1 / C} \frac{\varepsilon^{\mu}}{\alpha(\varepsilon)} \in(0,+\infty]$, where $C$ comes from (17). Let us mention that for any finite $\varepsilon>0$, $\alpha(\varepsilon)$ is finite, being the supremum of a USC multivalued function on a compact set. Fix $h \in(0, H)$. There exists $\varepsilon \in(0,1 / C)$ such that $h \leq \frac{\varepsilon^{\mu}}{\alpha(\varepsilon)}$. With this couple $(h, \varepsilon)$ we shall now prove the following fact.

Fact. For any sampling time $t_{k}$ such that $N\left(x\left(t_{k}\right)\right)^{-\kappa} \geq$ $\frac{\alpha(\varepsilon) h}{\varepsilon^{\mu}}$, we have

$$
N(x(t)) \leq \max \left\{\beta\left(N\left(x\left(t_{k}\right)\right), t-t_{k}\right) ; C \varepsilon N\left(x\left(t_{k}\right)\right)\right\}
$$

for all $t \in\left[t_{k}, t_{k+1}\right]$.

Remark first that if $x\left(t_{k}\right)=0$, then the asymptotic stability of the origin for the closed-loop system $\dot{x} \in$ $F(x, \Delta)$ implies that $x(t)=0$ for all $t \in\left[t_{k}, t_{k+1}\right]$ and the fact is proved. Now, to prove the fact when $x\left(t_{k}\right) \neq$ 0 , let us study the variations of $\Delta(t)$. We denote $s=$ $\ln \left(N\left(x\left(t_{k}\right)\right)\right)$. Consider

$$
\begin{aligned}
t^{*} & =\inf \left\{t \geq t_{k}: N(\Delta(t))>\varepsilon N\left(x\left(t_{k}\right)\right)\right\} \\
& =\inf \left\{t \geq t_{k}: V(\Delta(t))>\varepsilon^{\mu} V\left(x\left(t_{k}\right)\right)\right\}
\end{aligned}
$$

Thus on the interval $\left[t_{k}, t^{*}\right]$ we have $V(\Delta(t)) \leq$ $\varepsilon^{\mu} V\left(x\left(t_{k}\right)\right)$, which implies $V(\exp (-A s) \Delta(t)) \leq \varepsilon^{\mu}$ and $\exp (-A s) x\left(t_{k}\right) \in \mathbb{S}$ given that $N\left(x\left(t_{k}\right)\right)=e^{s}$. We have

$$
\begin{aligned}
V\left(\Delta\left(t^{*}\right)\right)= & \int_{t_{k}}^{t^{*}} \frac{d}{d t} V(\Delta(t)) d t \\
\leq & \left(t^{*}-t_{k}\right) \sup _{t \in\left[t_{k}, t^{*}\right]} \sup _{v \in-F(x, \Delta)}\left\|d_{\Delta(t)} V v\right\| \\
\leq & \left(t^{*}-t_{k}\right) \sup \left\{\left\|d_{\tilde{z}} V v\right\|: V(\exp (-A s) \tilde{z}) \leq \varepsilon^{\mu},\right. \\
& \exp (-A s) \tilde{y} \in \mathbb{S} \text { and } v \in F(\tilde{y}-\tilde{z}, \tilde{z})\}
\end{aligned}
$$

and from Assumption 14, it follows that

$$
\begin{aligned}
V\left(\Delta\left(t^{*}\right)\right) \leq & e^{(\kappa+\mu) s}\left(t^{*}-t_{k}\right) \sup \left\{\left\|d_{z} V v\right\|:\right. \\
& \left.V(z) \leq \varepsilon^{\mu}, y \in \mathbb{S} \text { and } v \in F(y-z, z)\right\} \\
\leq & N\left(x\left(t_{k}\right)\right)^{\kappa+\mu}\left(t^{*}-t_{k}\right) \alpha(\varepsilon)
\end{aligned}
$$

By continuity, we have $V\left(\Delta\left(t^{*}\right)\right)=\varepsilon^{\mu} V\left(x\left(t_{k}\right)\right)=$ $\varepsilon^{\mu} N\left(x\left(t_{k}\right)\right)^{\mu}$ which leads to

$$
\varepsilon^{\mu} N\left(x\left(t_{k}\right)\right)^{\mu} \leq N\left(x\left(t_{k}\right)\right)^{\kappa+\mu}\left(t^{*}-t_{k}\right) \alpha(\varepsilon)
$$

and then $t^{*}-t_{k} \geq \frac{\varepsilon^{\mu} N\left(x\left(t_{k}\right)\right)^{-\kappa}}{\alpha(\varepsilon)} \geq h$ that is $t^{*} \geq t_{k+1}$. Thus, for all $t \in\left[t_{k}, t_{k+1}\right]$ we have $N(\Delta(t)) \leq \varepsilon N\left(x\left(t_{k}\right)\right)$. Finally, inserting this inequality into (17) yields (18) and the fact is proved.

Step IV. The condition $N\left(x\left(t_{k}\right)\right)^{-\kappa} \geq \frac{\alpha(\varepsilon) h}{\varepsilon^{\mu}}$ is equivalent to $N\left(x\left(t_{k}\right)\right) \geq\left(\frac{\alpha(\varepsilon) h}{\varepsilon^{\mu}}\right)^{-\frac{1}{\kappa}}=\rho$. Hence $N\left(x\left(t_{k}\right)\right) \geq \rho$ ensures that $N\left(x\left(t_{k+1}\right)\right) \leq N(x(t))<N\left(x\left(t_{k}\right)\right)$ for all $t \in\left(t_{k}, t_{k+1}\right]$. However, the set $\left\{x \in \mathbb{R}^{n}: N(x)<\rho\right\}$ is not necessarily positively invariant. We therefore need to prove that, starting with $N\left(x\left(t_{k}\right)\right)<\rho$, a trajectory cannot go "too far" from the origin. Let us define, for $\omega \geq 0$

$$
\begin{array}{r}
\theta(\omega)=\sup \left\{\left\|d_{z} V v\right\|: V(z) \leq 1+\omega\right. \\
\quad V(y) \leq 1, v \in F(z, y-z)\} .
\end{array}
$$


Denoting $s=\frac{\ln (1+\omega)}{\mu}$, we get

$$
\begin{gathered}
\theta(\omega)=\sup \left\{\| d_{z} V v\right) \|: V(\exp (-A s) z) \leq 1 \\
\left.\quad V(\exp (-A s) y) \leq(1+\omega)^{-1} \text { and } v \in F(z, y-z)\right\} \\
=\sup \left\{\| d_{\exp (A s) \tilde{z} V v \|: V(\tilde{z}) \leq 1, V(\tilde{y}) \leq 1 /(1+\omega)}\right. \\
\quad \text { and } v \in F(\exp (A s) \tilde{z}, \exp (A s)(\tilde{y}-\tilde{z}))\}
\end{gathered}
$$

and from Assumption 14 it follows that

$$
\begin{gathered}
\theta(\omega)=e^{(\kappa+\mu) s} \sup \left\{\left\|d_{\tilde{z}} V v\right\|: V(\tilde{z}) \leq 1,\right. \\
\left.V(\tilde{y}) \leq(1+\omega)^{-1} \text { and } v \in F(\tilde{z}, \tilde{y}-\tilde{z})\right\} \\
=(1+\omega)^{\frac{\kappa+\mu}{\mu}} \sup \left\{\left\|d_{\tilde{z}} V v\right\|: V(\tilde{z}) \leq 1,\right. \\
V(\tilde{y}) \leq 1 /(1+\omega) \text { and } v \in F(\tilde{z}, \tilde{y}-\tilde{z})\} .
\end{gathered}
$$

Hence when $\omega \rightarrow+\infty$ we have $\theta(\omega) \sim \Omega \omega^{\frac{\kappa+\mu}{\mu}}$, with $\Omega=\sup \left\{\left\|d_{\tilde{z}} V v\right\|: V(\tilde{z}) \leq 1, v \in F(\tilde{z},-\tilde{z})\right\}$ and therefore $\frac{\omega}{\theta(\omega)} \rightarrow+\infty$, given that $\frac{\kappa+\mu}{\mu}<1$ since $\kappa<0$. Since the function $\omega \mapsto \frac{\omega}{\theta(\omega)}$ vanishes at $\omega=0$, there exists $\omega_{0}>0$ such that $\frac{\omega_{0}}{\theta\left(\omega_{0}\right)}=\frac{\varepsilon^{\mu}}{\alpha(\varepsilon)}$. Assume that $N\left(x\left(t_{k}\right)\right)<\rho$, i.e. $V\left(x\left(t_{k}\right)\right)<\rho^{\mu}$. Let us denote $t^{*}=$ $\inf \left\{t \geq t_{k}: V(x(t)) \geq\left(1+\omega_{0}\right) \rho^{\mu}\right\}>t_{k}$. Hence for all $t \in\left[t_{k}, t^{*}\right]$ we have $V(x(t)) \leq\left(1+\omega_{0}\right) \rho^{\mu}$. Therefore, we obtain

$$
\begin{aligned}
V\left(x\left(t^{*}\right)\right)= & V\left(x\left(t_{k}\right)\right)+\int_{t_{k}}^{t^{*}} \frac{d}{d t} V(x(t)) d t \\
\leq & \rho^{\mu}+\left(t^{*}-t_{k}\right) \sup \left\{\left\|d_{x(t)} V v\right\|:\right. \\
& \left.t \in\left[t_{k}, t^{*}\right], v \in F\left(x(t), x\left(t_{k}\right)-x(t)\right)\right\} \\
\leq & \rho^{\mu}+\left(t^{*}-t_{k}\right) \sup \left\{\left\|d_{z} V v\right\|: V(y) \leq \rho^{\mu}\right. \\
& \left.V(z) \leq\left(1+\omega_{0}\right) \rho^{\mu} \text { and } v \in F(z, y-z)\right\} \\
\leq & \rho^{\mu}+\left(t^{*}-t_{k}\right) \rho^{\kappa+\mu} \theta\left(\omega_{0}\right) .
\end{aligned}
$$

Also, by continuity, $V\left(x\left(t^{*}\right)\right)=\left(1+\omega_{0}\right) \rho^{\mu}$ and therefore after simplification and using $\rho=\left(\frac{\alpha(\varepsilon) h}{\varepsilon^{\mu}}\right)^{-\frac{1}{\kappa}}$ we get $\omega_{0} \leq\left(t^{*}-t_{k}\right)\left(\frac{\alpha(\varepsilon) h}{\varepsilon^{\mu}}\right)^{-1} \theta\left(\omega_{0}\right)$ and given that $\frac{\omega_{0}}{\theta\left(\omega_{0}\right)}=$ $\frac{\varepsilon^{\mu}}{\alpha(\varepsilon)}$ we find $t^{*}-t_{k} \geq h$. Finally, $t^{*} \geq t_{k+1}$ and then for all $t \in\left[t_{k}, t_{k+1}\right]$ we have $V(x(t)) \leq\left(1+\omega_{0}\right) \rho^{\mu}$, which implies $N(x(t)) \leq\left(1+\omega_{0}\right)^{1 / \mu} \rho$.

Let us summarize. We have three cases:

- if $N\left(x\left(t_{k}\right)\right)<\rho$ then we have $N(x(t)) \leq\left(1+\omega_{0}\right)^{1 / \mu} \rho$ for all $t \in\left[t_{k}, t_{k+1}\right]$;

- if $\rho \leq N\left(x\left(t_{k}\right)\right) \leq\left(1+\omega_{0}\right)^{1 / \mu} \rho$ then we still have $N(x(t)) \leq\left(1+\omega_{0}\right)^{1 / \mu} \rho$ for all $t \in\left[t_{k}, t_{k+1}\right]$;

- if $N\left(x\left(t_{k}\right)\right) \geq\left(1+\omega_{0}\right)^{1 / \mu} \rho$ then $N\left(x\left(t_{k}\right)\right)^{-\kappa} \geq \frac{\alpha(\varepsilon) h}{\varepsilon^{\mu}}$ and by the Fact of Step III we have

$$
N(x(t)) \leq \max \left\{\beta\left(N\left(x\left(t_{k}\right)\right), t-t_{k}\right) ; C \varepsilon N\left(x\left(t_{k}\right)\right)\right\}
$$

for all $t \in\left[t_{k}, t_{k+1}\right]$.

We get from the first two points that the set $\{N(x) \leq$ $\left.\left(1+\omega_{0}\right)^{1 / \mu} \rho\right\}$ is positively invariant. Hence, if $N\left(x_{0}\right)>$ $\left(1+\omega_{0}\right)^{1 / \mu} \rho$, denoting $k^{*}=\max _{k \geq 0}\left\{N\left(x\left(t_{k}\right)\right) \geq(1+\right.$ $\left.\left.\omega_{0}\right)^{1 / \mu} \rho\right\}$, for all $0 \leq k \leq k^{*}$, we have $N\left(x\left(t_{k}\right)\right) \geq$ $\left(1+\omega_{0}\right)^{1 / \mu} \rho$. Therefore the third point gives us that $N\left(x\left(t_{k+1}\right)\right) \leq N\left(x\left(t_{k}\right)\right)$ for all $0 \leq k \leq k^{*}$ and thus $N\left(x\left(t_{k}\right)\right)<\bar{N}\left(x_{0}\right)$ for all $0<k \leq k^{*}$. Using again the shape of the function $\beta$, for $0 \leq k<k^{*}$ and $t \in\left[t_{k}, t_{k+1}\right]$ we get

$$
\begin{aligned}
N(x(t)) & \leq \max \left\{\beta\left(N\left(x\left(t_{k}\right)\right), \eta\right) ; C \varepsilon N\left(x\left(t_{k}\right)\right)\right\} \\
& \leq N\left(x\left(t_{k}\right)\right) \max \left\{\beta\left(1, \eta N\left(x\left(t_{k}\right)\right)^{\kappa}\right) ; C \varepsilon\right\} \\
& \leq N\left(x\left(t_{k}\right)\right) \max \left\{\beta\left(1, \eta N\left(x_{0}\right)^{\kappa}\right) ; C \varepsilon\right\} \\
& \leq N\left(x\left(t_{k}\right)\right) q
\end{aligned}
$$

where $q=\max \left\{\beta\left(1, \eta N\left(x_{0}\right)^{\kappa}\right) ; C \varepsilon\right\}<1$, given that $\beta \in \mathcal{K} \mathcal{L}$ and $\beta(r, 0)=r$. Therefore

$$
N(x(t)) \leq \max \left\{N\left(x_{0}\right) q^{k} ;\left(1+\omega_{0}\right)^{1 / \mu} \rho\right\}, t \in\left[t_{k}, t_{k+1}\right]
$$

and we get the global asymptotical stability of the set $\left\{x \in \mathbb{R}^{n}: N(x) \leq\left(1+\omega_{0}\right)^{1 / \mu} \rho\right\}=\left\{x \in \mathbb{R}^{n}: N(x) \leq\right.$ $\left.\xi h^{-1 / \kappa}\right\}$.

Remark 20 The assumptions taken on the sampling sequence $\left(t_{k}\right)$ imply that $\left(t_{k}\right)$ is monotonously increasing and does not admit an accumulation point.

Note that the result holds for any homogeneous norm. Changing the homogeneous norm would only change the number $\xi$ in the definition of the asymptotically set $\mathcal{N}$.

\section{Application to the double integrator}

Consider the double integrator (9) and for $t \in\left[t_{k}, t_{k+1}\right)$, we select the control law to be

$$
u\left(x\left(t_{k}\right)\right)=-k_{1} \operatorname{sign}\left(x_{1}\left(t_{k}\right)\right)-k_{2} \operatorname{sign}\left(x_{2}\left(t_{k}\right)\right)
$$

with $k_{1}>k_{2}$. For the simulations, we take $k_{1}=5$ and $k_{2}=2.5$. We choose a constant sampling period

$$
t_{k}=k T, \quad T>0, k \in \mathbb{N}
$$

in order to use well known results about linear systems having a constant sampling period. Indeed, we know that the closed-loop linear sampled-data system (9) under the linear controller $u\left(x\left(t_{k}\right)\right)=-k_{1} x_{1}\left(t_{k}\right)-k_{2} x_{2}\left(t_{k}\right)$ with the samplings (20) is asymptotically stable if and only if the matrix $\Lambda(T)$ of its associated linear difference equation is Schur $[34,35]$. For the double integrator with $k_{1}=5$ and $k_{2}=2.5, \Lambda(T)$ is Schur if and only if $T<T_{\text {Schur }}=0.8 \mathrm{~s}$. However, Theorem 19 yields that, 
for any $[2,1]$-homogeneous norm, there exists $\xi>0$ such that the set $\left\{x \in \mathbb{R}^{n}: N(x) \leq \xi T\right\}$ is globally asymptotically stable for the nonlinear closed-loop system (9)(19)-(20). In Figure 1, the asymptotically stable sets are drawn for different constant samplings $T$ larger than $T_{\text {Schur }}=0.8 \mathrm{~s}$.

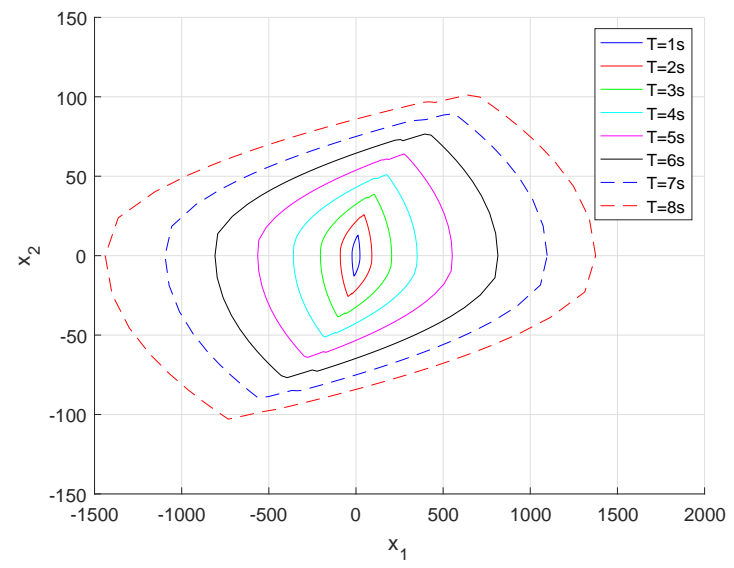

Fig. 1. Asymptotically stable sets for different values of $T$

\section{Conclusion}

In this article, we have extended the results about stability of homogeneous sampled-data systems to discontinuous systems. In particular, we have designed a discontinuous sampled controller for the double integrator. The robustness with respect to model uncertainties of discontinuous controllers compared with the continuous ones in the sampled-data context is an interesting issue for futures works.

\section{References}

[1] W. Zhang, M. S. Branicky, S. M. Phillips, Stability of networked control systems, IEEE Control Systems 21 (1) (2001) 84-99.

[2] G. C. Walsh, H. Ye, L. G. Bushnell, Stability analysis of networked control systems, IEEE Transactions on Control Systems Technology 10 (3) (2002) 438-446.

[3] D. Nešić, A. R. Teel, Input-output stability properties of networked control systems, IEEE Transactions on Automatic Control 49 (10) (2004) 1650-1667.

[4] N. W. Bauer, P. J. H. Maas, W. P. M. H. Heemels, Stability analysis of networked control systems: A sum of squares approach, Automatica 48 (8) (2012) 1514-1524.

[5] M. C. F. Donkers, W. P. M. H. Heemels, N. Van De Wouw, L. Hetel, Stability analysis of networked control systems using a switched linear systems approach, IEEE Transactions on Automatic Control 56 (9) (2011) 2101-2115.

[6] D. Nešić, A. R. Teel, A framework for stabilization of nonlinear sampled-data systems based on their approximate discrete-time models, IEEE Transactions on automatic control 49 (7) (2004) 1103-1122.
[7] H. Fujioka, Stability analysis of systems with aperiodic sample-and-hold devices, Automatica 45 (3) (2009) 771-775.

[8] K. Liu, E. Fridman, L. Hetel, Networked control systems: A time-delay approach, in: European Control Conference, Strasbourg, France, 2014, pp. 1434-1439.

[9] F. Mazenc, M. Malisoff, T. N. Dinh, Robustness of nonlinear systems with respect to delay and sampling of the controls, Automatica 49 (6) (2013) 1925-1931.

[10] A. Seuret, A novel stability analysis of linear systems under asynchronous samplings, Automatica 48 (1) (2012) 177-182.

[11] H. Omran, L. Hetel, J.-P. Richard, F. Lamnabhi-Lagarrigue, On the stability of input-affine nonlinear systems with sampled-data control, in: European Control Conference, Zürich, Switzerland, 2013, pp. 2585-2590.

[12] A. Bemporad, M. Heemels, M. Johansson, Networked control systems, Vol. 406 of Lecture Notes in Control and Information Sciences, Springer, 2010.

[13] D. Nešić, A. R. Teel, D. Carnevale, Explicit computation of the sampling period in emulation of controllers for nonlinear sampled-data systems, IEEE Transactions on Automatic Control 54 (3) (2009) 619-624.

[14] V. Zubov, On ordinary differential equations with generalized homogeneous right-hand sides (in Russian), Izvestiya vuzov, Matematika 1 (2) (1958) 80-88.

[15] L. P. Rothschild, E. M. Stein, Hypoelliptic differential operators and nilpotent groups, Acta Mathematica 137 (1) (1976) 247-320.

[16] S. P. Bhat, D. S. Bernstein, Geometric homogeneity with applications to finite-time stability, Mathematics of Control, Signals and Systems 17 (2) (2005) 101-127.

[17] M. Kawski, Geometric homogeneity and stabilization, in: Nonlinear Control Systems Design, Elsevier, 1995, pp. 147152.

[18] E. Moulay, Stability and stabilization of homogeneous systems depending on a parameter, IEEE Transactions on Automatic Control 54 (6) (2009) 1382-1385.

[19] H. Hermes, Nilpotent and high-order approximations of vector field systems, SIAM review 33 (2) (1991) 238-264.

[20] E. Bernuau, E. Moulay, P. Coirault, Stability of homogeneous nonlinear systems with sampled-data inputs, Automatica 85 (2017) 349-355.

[21] Y. V. Orlov, Discontinuous systems: Lyapunov analysis and robust synthesis under uncertainty conditions, Communications and Control Engineering, Springer, 2008.

$[22]$ E. Bernuau, D. Efimov, W. Perruquetti, A. Polyakov, On an extension of homogeneity notion for differential inclusions, in: European Control Conference, Zürich, Switzerland, 2013, pp. 2204-2209.

[23] E. Bernuau, D. Efimov, W. Perruquetti, Robustness of homogeneous and homogeneizable differential inclusions, in: Advances in Variable Structure Systems and Sliding Mode ControlTheory and Applications, Springer, 2018, pp. 39-56.

[24] X. Han, E. Fridman, S. K. Spurgeon, Sampled-data sliding mode observer for robust fault reconstruction: A time-delay approach, Journal of the Franklin Institute 351 (4) (2014) $2125-2142$.

[25] T. Nguyen, W.-C. Su, Z. Gajic, Output feedback sliding mode control for sampled-data systems, IEEE Transactions on Automatic Control 55 (7) (2010) 1684-1689.

[26] K. D. Young, V. I. Utkin, U. Ozguner, A control engineer's guide to sliding mode control, IEEE Transactions on Control Systems Technology 7 (3) (1999) 328-342. 
[27] E. Cruz-Zavala, J. A. Moreno, Homogeneous high order sliding mode design: A Lyapunov approach, Automatica 80 (2017) 232-238.

[28] A. Filippov, Differential Equations with Discontinuous Righthand Sides, Vol. 18 of Mathematics and Its Applications, Springer, 1988.

[29] E. D. Sontag, Y. Wang, On characterizations of the inputto-state stability property, Systems \& Control Letters 24 (5) (1995) 351-359.

[30] A. Bacciotti, L. Rosier, Liapunov functions and stability in control theory, 2nd Edition, Communications and Control Engineering, Springer, 2005.

[31] E. Bernuau, D. Efimov, W. Perruquetti, Robustness of homogeneous and locally homogeneous differential inclusions, in: European Control Conference, 2014, pp. 2624-2629.

[32] E. Bernuau, Robustness and stability of nonlinear systems: a homogeneous point of view, Ph.D. thesis, Ecole Centrale de Lille (2013).

URL https://tel.archives-ouvertes.fr/tel-00917798/

[33] Y. Orlov, Finite time stability and robust control synthesis of uncertain switched systems, SIAM Journal on Control and Optimization 43 (4) (2004) 1253-1271.

[34] L. Hetel, C. Fiter, H. Omran, A. Seuret, E. Fridman, J.-P Richard, S. I. Niculescu, Recent developments on the stability of systems with aperiodic sampling: an overview, Automatica 76 (2017) 309-335.

[35] S. Maalej, C. Fiter, L. Hetel, J.-P. Richard, State-dependent sampling for linear time invariant systems: a discrete time analysis, in: 20th IEEE Mediterranean Conference on Control \& Automation, 2012, pp. 1129-1134. 